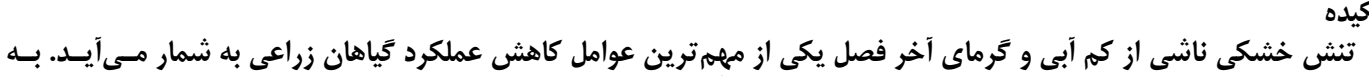

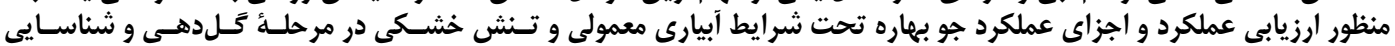

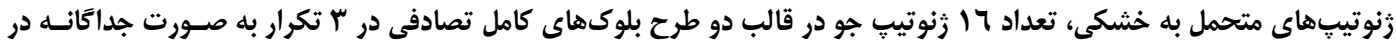

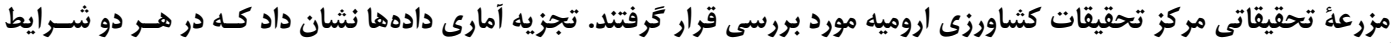

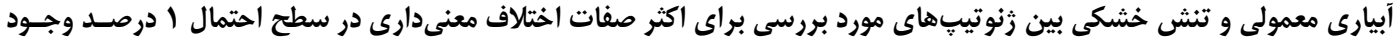

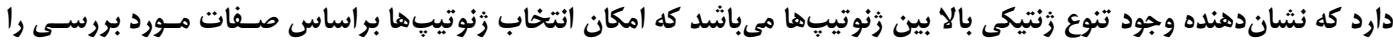

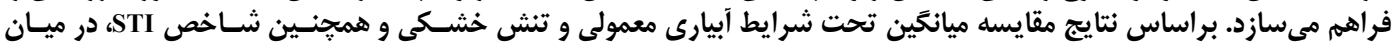

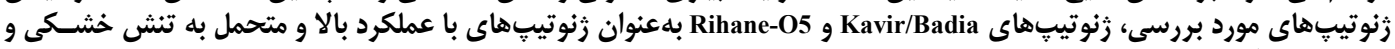

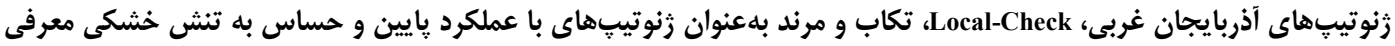

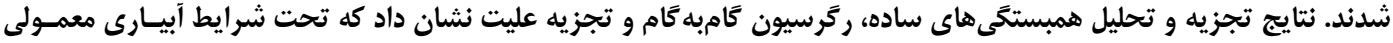

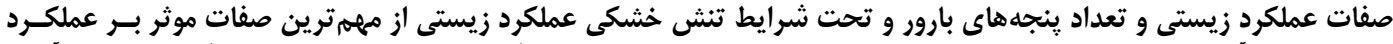

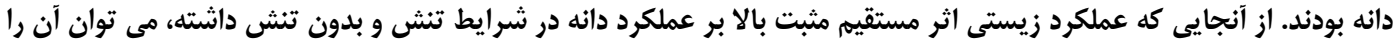

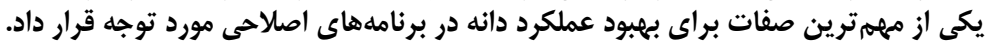

وازههاى كليدى: رَّرسيون كَامبه كَام، تجزيه عليت، تحليل همبستكى، تنش خشكى، جو

استفاده قرار گرفته است. فرناندز (N) شاخص حساسيت به

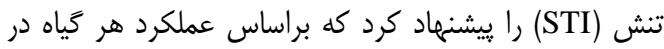

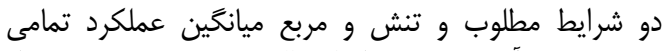

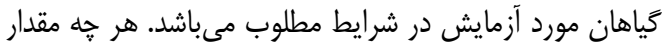
STI

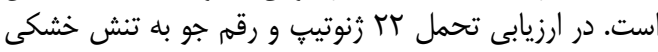

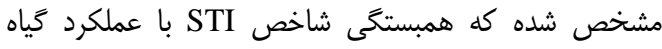

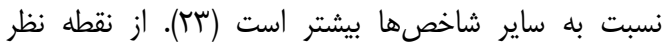

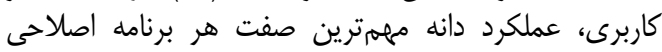

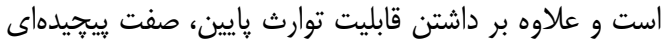

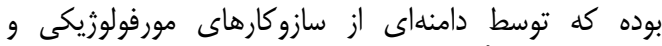

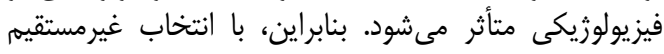

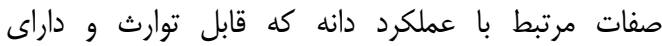

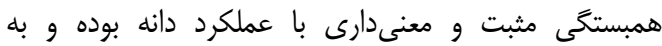

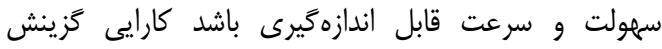

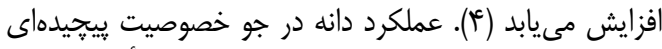

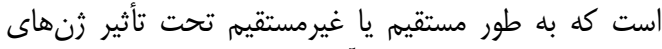

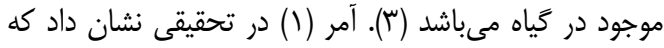

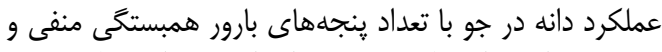

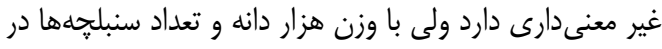

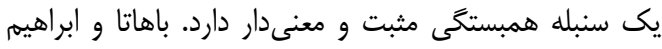

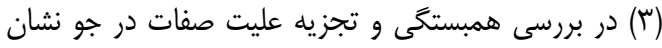

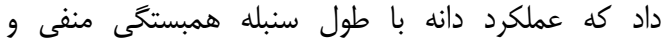
غيرمعنى دار و با تعداد ينجه بارور همبستخى منفى هنى و معنى منى دار
عملكرد گياهان زراعى تحت تأثير ساختار زنتيكى گياه،

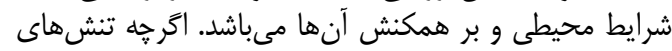

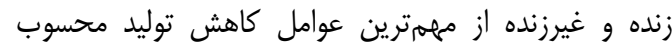

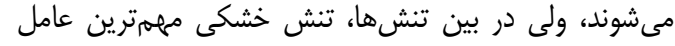

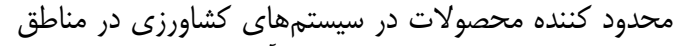

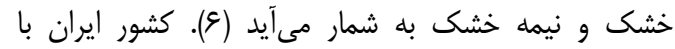

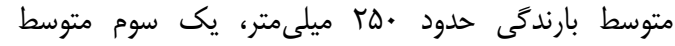

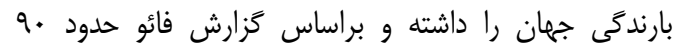

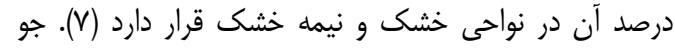

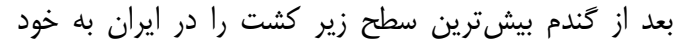

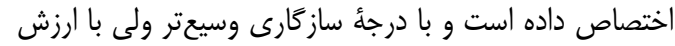

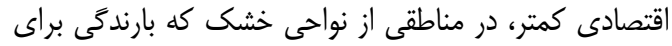

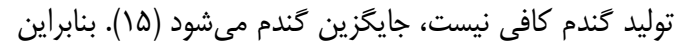

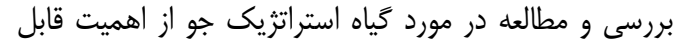

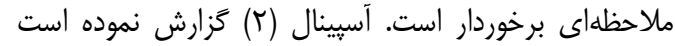

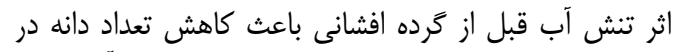

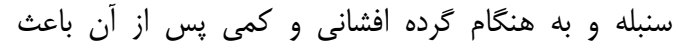

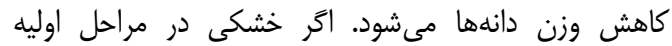

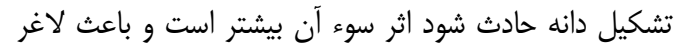

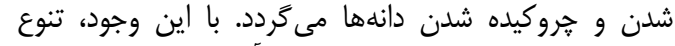

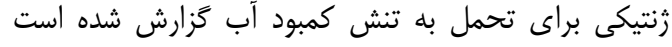
(19،IV) زراعى نسبت به تنشهاى محيطى توسط محققين مورد مختى ماتى 
فصل بر روى صفات فنولوزيك، عملكرد و اجزاى عملكرد و تصوي

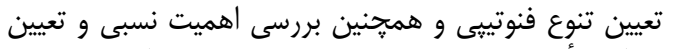

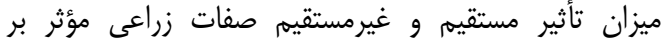

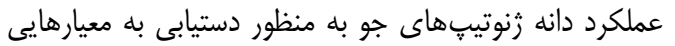

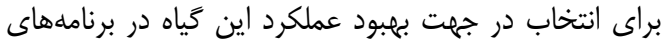
بلنزادى انجام گَرفت.

\section{مواد و روشها}

به منظور ارزيابى عملكرد و اجزاى عملكرد جو إيار بهاره

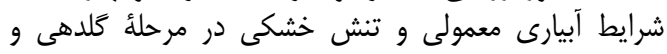

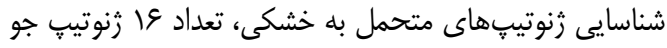

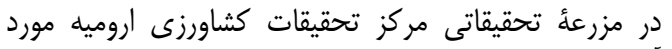

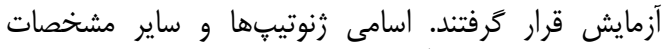
مربوطه در جدول (1) آورده شده است.
دارد. همجنين، در اين مطالعه عملكرد دانه با صفات ارتفاع

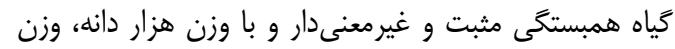

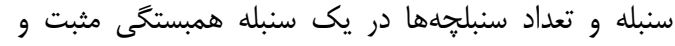

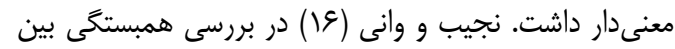

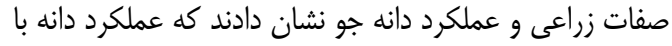

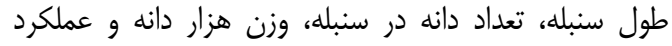

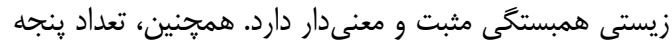

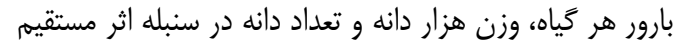

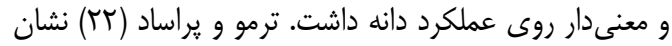

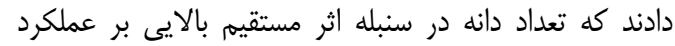

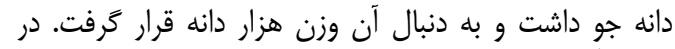

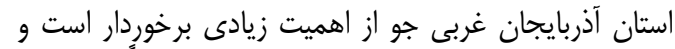

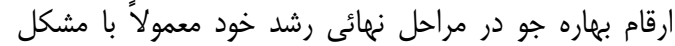

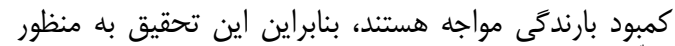

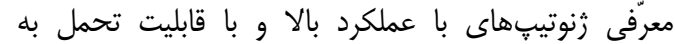
خشكى در اواخر دوره رشد، بررسى اثرات بتش بنش خشكى

Table 1. Information of investigated barley genotypes

جدول |- اطلاعات زنوتيِهاى جو مورد بررسى

\begin{tabular}{|c|c|c|}
\hline تيب سنبله & نام زنوتيٍ & شماره \\
\hline ا تش رديفه & Local- Check & 1 \\
\hline شش رديفه & Rihane & $r$ \\
\hline ش رديفه & Rihane/Alger- Union & r \\
\hline ش رديفه & Atlas 46/Kavir & f \\
\hline شش رديفه & Gorgan/CM 67/Pro/Svo & $\Delta$ \\
\hline ش ش رديفه & Suifu/Walfajre & 8 \\
\hline ش ش رديفه & QC 2.17/D7/Bgs & v \\
\hline 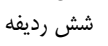 & Kavir/Badia & $\wedge$ \\
\hline ش ش رديفه & Rihane-O5 & 9 \\
\hline شش رديفه & AS 46/ Ahtaz-ZAD & 1. \\
\hline ا دو رديفه & Landrace (West Azarbaijan) & 11 \\
\hline دو رديفه & Landrace (Tekab) & ir \\
\hline دو رديفه & Landrace (Marand) & r \\
\hline دو رديفه & Hebe & If \\
\hline دو ديفه & Union 300-4 & 10 \\
\hline دو رديفه & Landrace (Salmas) & 18 \\
\hline
\end{tabular}

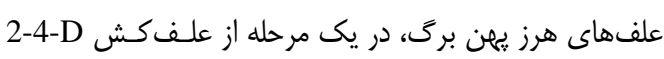

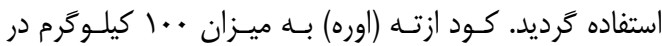

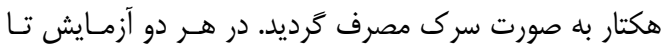

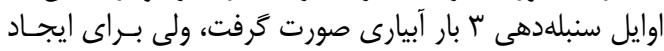

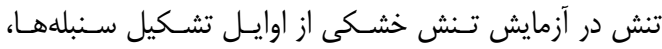

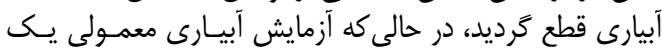

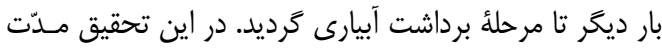

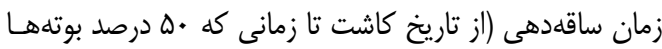

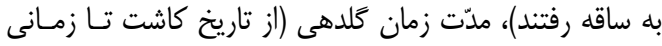

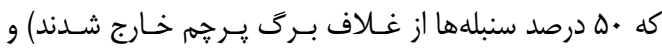

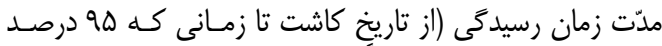

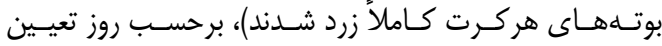

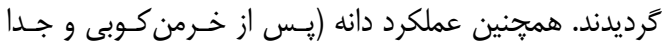

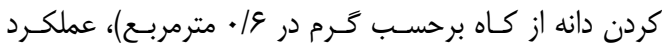

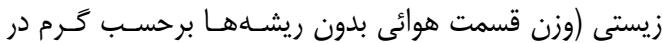

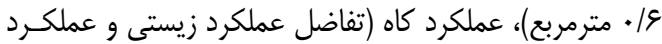

تحقيق حاضر در قالب دو آزمايش جداكانئ آبيارى معمولى

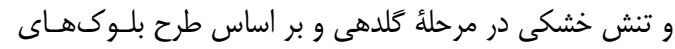

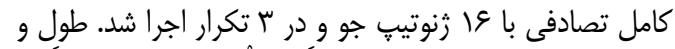

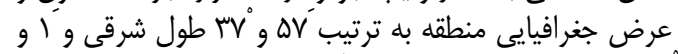

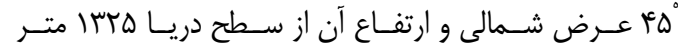

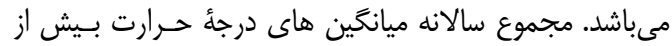

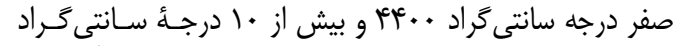

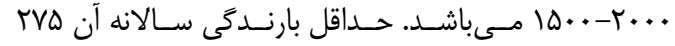

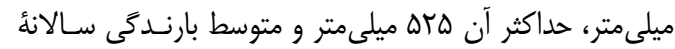

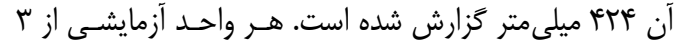

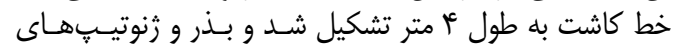

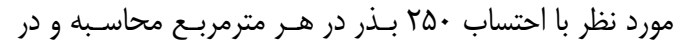

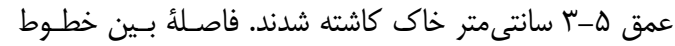

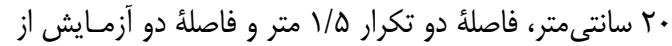

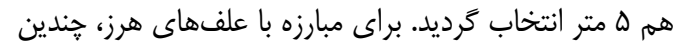

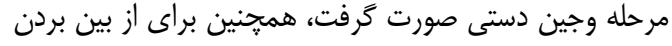




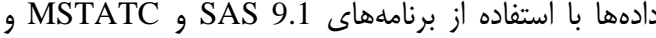

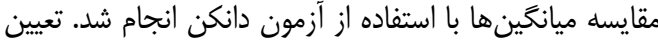

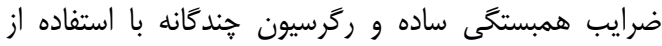

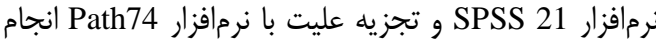

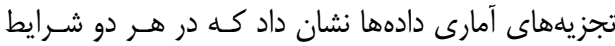

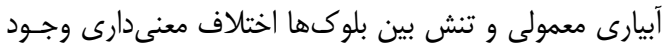

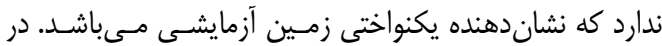

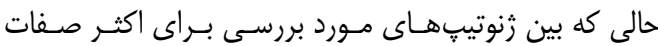

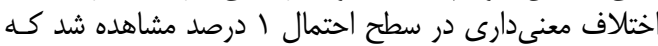

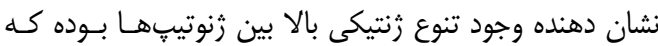

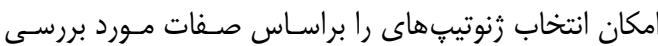

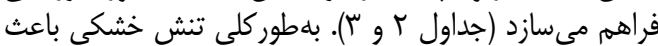

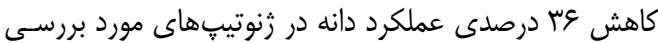

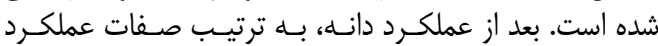

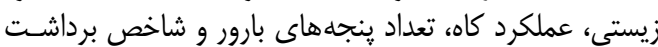

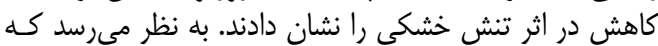

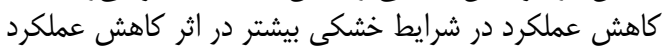

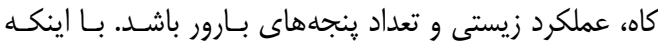

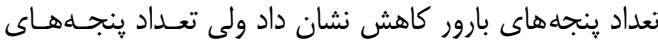

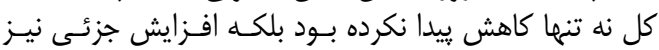

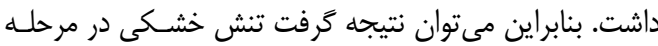

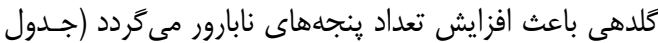

دانه)، شـاخص برداشـت (نسـبت عملكـرد دانـه بــهـ عملكـرد

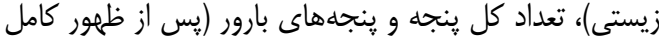

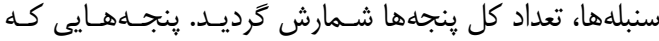

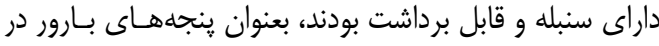

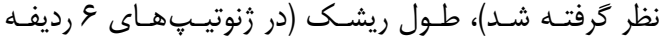

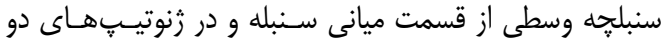

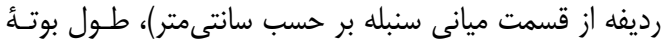

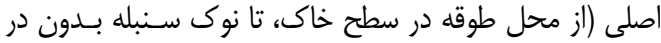

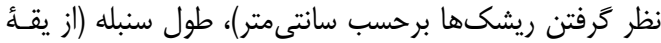

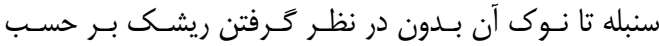

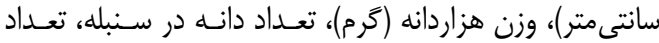

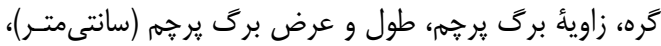

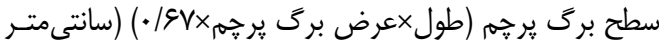

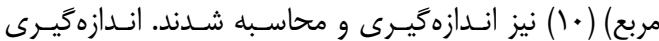

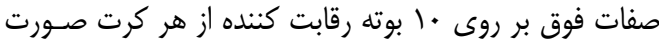

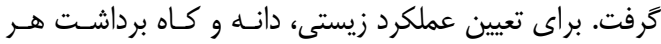

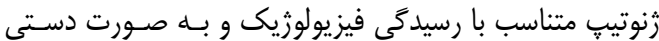

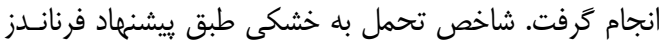

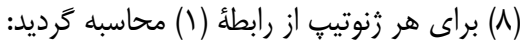

$$
S T I=\frac{\left(Y_{S}\right)\left(Y_{P}\right)}{\left(\bar{Y}_{P}\right)^{2}}
$$

كه در آن STI شاخص تحمل تنش، كآ

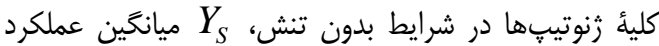
هر زنوتيب در شرايط تنش و و

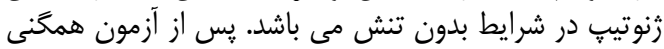
واريانسها و نرمال بودن توزيع اشتباهات آزمايشى، تجزيه آزيه

Table 2. Analysis of variance of investigated traits in 16 barley genotypes under normal conditions

\begin{tabular}{|c|c|c|c|c|c|c|c|c|c|c|c|}
\hline $\begin{array}{c}\text { تغييرات } \\
\text { (S.O.V.) }\end{array}$ & $\begin{array}{l}\text { آزادى درجه } \\
\text { (df) }\end{array}$ & وزن .... دانه & عملكرد دانه & $\begin{array}{c}\text { عملكرد كاه } \\
\text { (SY) }\end{array}$ & $\begin{array}{c}\text { عملكرد زيستى } \\
\text { (BY) }\end{array}$ & $\begin{array}{c}\text { شاخص برداشت } \\
\text { (HI) }\end{array}$ & تعداد دانه در & هاى بارور & 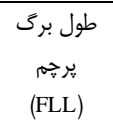 & عرض برى & سطح برى \\
\hline تكرار & $r$ &.$/ r F q q^{\mathrm{ns}}$ & $r \Delta V \cdot N / q r^{n s}$ & $190 \% 9 \cdot / 9 . \mathrm{ns}$ & $r V r V r r / q)^{\mathrm{ns}}$ & $r / r T \Delta r^{\mathrm{ns}}$ & V/larrns & $. / \cdot 4 \|^{n s}$ & $\cdot / \cdot v^{\prime} 9^{\mathrm{ns}}$ &.$/ .19^{\mathrm{ns}}$ & I/VGYV ${ }^{\mathrm{ns}}$ \\
\hline تنش & 10 & QNTAKT ${ }^{* * *}$ & $1.4 \%$. $9 / 9 q^{* *}$ & TITNGVGN/rF'** & TATKFA. & $\mid r V / . . k r^{* * *}$ & $r V Y / r V q 9^{* *}$ &.$/ 4 \cdot \lambda r^{* * *}$ & $\mid \varepsilon / . \kappa r \sigma^{* *}$ &.$/ T I F f^{* * *}$ & $\| N / r \cdot \sigma^{* *}$ \\
\hline اشتباه & r. & س & IATهN/AD & VNEATNAT & rGqVQ / $/<q$ & M/яqтq & ه/A & . Frf & 1/1994 & הזr. & سوוצ/ץ \\
\hline ضغريب & - & & $N N^{\mu}$ & $\mid F / \Delta \Delta$ & GIAT & $9 / 1 V$ & $q / \pi q$ & $1 . / 41$ & $V / * t^{c}$ & $\mid F / A T$ & $1 T / 19$ \\
\hline
\end{tabular}

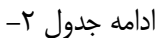

Table 2. Continued

\begin{tabular}{|c|c|c|c|c|c|c|c|c|c|c|c|c|}
\hline $\begin{array}{c}\text { منابع تغييرات } \\
\text { (S.O.V.) }\end{array}$ & $\begin{array}{c}\text { درجه آزادى } \\
\text { (df) }\end{array}$ & روز تا ساقه & $\begin{array}{l}\text { روز تا كلدهى (DF) } \\
\text { ركى }\end{array}$ & 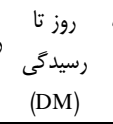 & طول ميانگُره & $\begin{array}{c}\text { طول سنبله } \\
\text { (SL) }\end{array}$ & 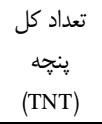 & $\begin{array}{c}\text { طول ريشك } \\
\text { (AL) }\end{array}$ & $\begin{array}{c}\text { ارتفاع بوته } \\
\text { (PH) }\end{array}$ & 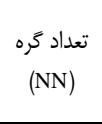 & 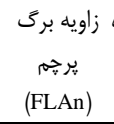 & $\begin{array}{c}\text { طول ميانغره از انتها } \\
\text { (FIN) }\end{array}$ \\
\hline تكرار & r & $\Delta / \cdot r \cdot \wedge^{\text {ns }}$ & $r / T V \cdot \lambda^{n s}$ & r/DGTQ ns & $.1 .9 Y \Delta^{\mathrm{ns}}$ & $. / . \uparrow q .+\mathrm{ns}$ & $\%$ rqns &.$/ \cdot 4 r^{\mathrm{ns}}$ & $\Delta / \mathcal{F}^{\top} \backslash \Lambda^{\mathrm{nS}}$ & $\cdot / 1 \cdot 98^{\mathrm{ns}}$ & $V / \Delta q \cdot V^{n s}$ & V/DAT. ns \\
\hline تنش & 10 & $9 / .911^{* * *}$ & $\Delta F / \Lambda F F F^{* * *}$ & 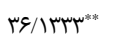 & r/৭น ז"*** & $F / N G \mid Q^{* * *}$ & T/Q9).*** & $r / g\|\| r^{* *}$ & rI/צqৎqns & . & $91 / 4{ }^{\prime}$ & $|\Delta / \Delta| r . * *$ \\
\hline اشتباه & r. & r/DQRe & IT/AFNE & . IDFTA & - IALYE & $\cdot / r \Lambda \cdot F$ & . /FAץ & ع & TY/TEGV &.$/$ TVI & F/DVIT & T/TLIV \\
\hline ضريب تغييرات & - & $r / q$. & D/9 & $\cdot / \mathrm{Nq}$ & SNT & $g / N^{\mu}$ & १/VQ & $V / 99$ & $N \cdot 9$ & $10 / 9 \pi$ & $9 / 1$ & १/द. \\
\hline
\end{tabular}


جدول זـ- تجزيه واريانس صفات مورد بررسى در 1 ازنوتيب جو تحت شرايط تنش خشكى Table 3. Analysis of variance of investigated traits in 16 barley genotypes under drought stress conditions

\begin{tabular}{|c|c|c|c|c|c|c|c|c|c|c|c|}
\hline $\begin{array}{c}\text { منابع تغييرات } \\
\text { (S.O.V.) }\end{array}$ & $\begin{array}{c}\text { درجه آزادى } \\
\text { (df) }\end{array}$ & وزن ... إنه & $\begin{array}{c}\text { عملكرد دانه } \\
\text { (GY) }\end{array}$ & $\begin{array}{c}\text { عملكرد كاه } \\
\text { (SY) }\end{array}$ & $\begin{array}{c}\text { عملكرد زيستى } \\
\text { (BY) }\end{array}$ & $\begin{array}{c}\text { شاخص برداشت } \\
\text { (HI) }\end{array}$ & تعداد دانه در & 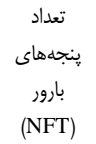 & $\begin{array}{l}\text { طول برى } \\
\text { يرجم } \\
\text { (FLL) }\end{array}$ & 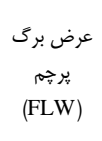 & $\begin{array}{c}\text { سطح برى } \\
\text { سرحم) } \\
\text { (FLAr) }\end{array}$ \\
\hline تكرار & r & . $/$ QTYY ns & 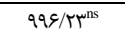 & $\Delta q 9 V T / \Lambda^{n s}$ & $9011 \mathrm{r} / \Lambda r^{\mathrm{ns}}$ & . $/ \wedge r \Delta \cdot{ }^{\mathrm{ns}}$ & g/FIrrns &.$/ \cdot 1 \wedge \mathrm{m}^{\mathrm{ns}}$ & $r / F^{E} \Delta F Q^{\mathrm{nS}}$ & $. / . .9 .^{\mathrm{ns}}$ & $r / V I r \Lambda^{n s}$ \\
\hline تنش & 10 & $1 \cdot T / 1 \cdot 11^{* * *}$ & FMMTVV/VQ** & $|V| r V \Delta q Y /\left.\wedge\right|^{* *}$ & $M T r \cdot q T \cdot 1 / 9 D^{* * *}$ & ITE/MYYA** & $r \cdot r / \Delta \backslash \& Y^{* * *}$ & $\cdot / \mathcal{F} \wedge \Delta \mathcal{F}^{* * *}$ & |V/grrqu* & $\cdot / \wedge \triangle Q^{* * *}$ & $59 / 194 \Lambda^{*}$ \\
\hline اشتباه & r. & $\Delta / r F \cdot \Delta$ & $9 \Delta V \cdot / Q S$ & $|V \cdot|. r / \Delta r$ & ISTAQS/Tr & D/DVYG & $1 . / 1 r v \Delta$ & $.1 . \mathrm{Fr}$ & $r / M \cdot \Delta$ &.$/$ HA $^{\prime}$ & $V / \triangle Q I V$ \\
\hline ضريب تغييرات & - & $F / \Delta \Lambda$ & $V / \& \&$ & $N \cdot F$ & $9 / 4)$ & $\mid r / N F$ & $\mid T / \pi T$ & $|r / 4|$ & $11 / \cdot v$ & $10 / \mathrm{v}$. & $19 / M$ \\
\hline
\end{tabular}

Table 3. Continued

ادامه جدول r

\begin{tabular}{|c|c|c|c|c|c|c|c|c|c|c|c|c|}
\hline $\begin{array}{c}\text { منابع تغييرات } \\
\text { (S.O.V.) }\end{array}$ & $\begin{array}{c}\text { درجه آزادى } \\
\text { (df) }\end{array}$ & روز تا ساقه & $\begin{array}{c}\text { روز تا كلدهى (DF) } \\
\text { (DF }\end{array}$ & $\begin{array}{c}\text { روز تا رسيدگى } \\
\text { (DM) }\end{array}$ & $\begin{array}{c}\text { ميانكره } \\
\text { دوم از } \\
\text { دولتها } \\
\text { (LSIN) }\end{array}$ & $\begin{array}{c}\text { طول سنبله } \\
\text { (SL) }\end{array}$ & 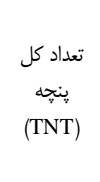 & $\begin{array}{c}\text { طول ريشك } \\
\text { (AL) }\end{array}$ & $\begin{array}{c}\text { ارتفاع بوته } \\
\text { (PH) }\end{array}$ & $\begin{array}{c}\text { تعداد گره } \\
\text { (NN) }\end{array}$ & زاويه برى & $\begin{array}{c}\text { ميانكره اول } \\
\text { از انتها } \\
\text { (FIN) }\end{array}$ \\
\hline تكرار & $r$ & $\cdot / \Lambda \wedge \vee Q^{\mathrm{ns}}$ & $19 / 1 F \Delta \Lambda^{*}$ & $1 / r \cdot \Lambda^{\mathrm{ns}}$ & 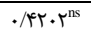 & $\cdot / r \Delta V^{e n s}$ & $\cdot / \cdot \Lambda r^{\mathrm{ns}}$ & . TquY ns & $\Delta / \varsigma \& 9$. ns & $\cdot / T Y \backslash Q^{\mathrm{ns}}$ & T/TFAYns & $r / V^{\mu} \Lambda . n s$ \\
\hline تنش & 10 & $\mid r / \Delta q \wedge \varepsilon^{*}$ & $\Delta N / 4 r \wedge q^{* * *}$ & rI/VYris & $1 / \xi 94 \wedge^{\mathrm{ns}}$ & g/TVYr** & $r / \Delta \xi I V^{* * *}$ & $\Delta / / F V \cdot{ }^{* * *}$ & $F \Delta / r \Lambda \cdot r^{* * *}$ & & ITNAMFV** & $q /$ q . *** \\
\hline اشتباه & $r$. & $\Delta / \cdot 9 \wedge \varepsilon$ & r/ArEV & ודזקופו & $1 / r \cdot 1 r$ & $.1 .9 V^{c}$ & $\cdot / \Lambda \varepsilon \cdot r$ & . MtrAN & $10 / 9 \& 90$ & $\cdot / r \cdot \Delta 9$ & $r / \Delta 111$ & $r / \Upsilon \subset \wedge 1$ \\
\hline ضريب تغييرات & - & $\Delta / \& T$ & $\% / \wedge$ & $1 / r V$ & $N \cdot r$ & r/qA & $1.1 . r$ & $r / \cdot 1$ & $\mathrm{~V} / \cdot \cdot$ & $1 r / \cdot 9$ & V/Ar & $N / 4$. \\
\hline
\end{tabular}

جدول عأ- ميانخين و درصد تغييرات صفات مورد بررسى در زنوتيبهاى جو تحت شرايط آبيارى معمولى و تنش خشكى

Table 4. Mean and percentage of changes of investigated traits in barley genotypes under normal and drought stress

\begin{tabular}{|c|c|c|c|c|}
\hline Traits & صفات & آبيارى معمولى & تنش خشكى & درصد تغييرات \\
\hline $1000 \mathrm{GW}$ & وزن هزار دانه (كرم) & $\Delta \backslash / \Gamma$. & $\Delta \cdot / f^{c} f$ & $1 / 9 V$ \\
\hline GY & عملكرد دانه (كرم) & $199 \mathrm{~N} \cdot \mathrm{r}$ & $1 \cdot 1 \varepsilon / 9 \vee$ & ५ \\
\hline SY & عملكرد كاه (كرم) & $9 \cdot 90 / f^{6}$ & $011 \% \cdot / 91$ & ID/AT \\
\hline BY & عملكرد زيستى (كرم) & VET.Mr & grav/M & G/V/V \\
\hline $\mathrm{HI}$ & شاخص برداشت (\%) & $r \cdot / \Lambda V$ & $19 / 40$ & $9 / \mathrm{V} \wedge$ \\
\hline NGS & تعداد دانه در سنبله & ra/NT & ra/AT & $-* / \mu r$ \\
\hline NFT & تعداد ينجه بارور & 1/98 & I/VY & س 1/.1 \\
\hline FLL & طول برگ يرجم (سانتى متر) & $1 \Delta / \Delta \Delta$ & س/D/ & I/ft \\
\hline FLW & عرض برى يرجم (سانتىمتر) & I/T & $1 / T \Delta$ & 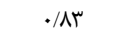 \\
\hline FLAr & سطح برگ پرخم (سانتى متر مربع) & $\mid r / T V$ & 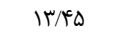 &.$- / 1 \wedge$ \\
\hline DS & تعداد روز تا •ه درصد ساقهدهى & $F i / T q$ & $|f| / 9 q$ &.$- / 99$ \\
\hline DF & تعداد روز تا •ه درصد كلدهى & 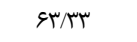 & GT/DF & 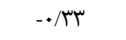 \\
\hline DM & تعداد روز تا •ه درصد رسيدگى & $9 \Delta / \cdots$ & $9 \% / T \vee$ & I/AT \\
\hline LSIN & طول ميانكره دوم از انتها (سانتىمتر) & ir/ne & Ir/gV & $\cdot / \Delta$ \\
\hline SL & طول سنبله (سانتىمتر) & V/AV & V/AF & $\cdot / \mathrm{r}$ \\
\hline TNT & تعداد هنجه كل & ए/१८ & 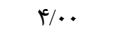 & $-1 / T \Lambda$ \\
\hline $\mathrm{AL}$ & طول ريشك (سانتىمتر) & $11 / v 9$ & $\| / N$ & tet \\
\hline $\mathrm{PH}$ & ارتفاع بوته (سانتىمتر) & $\Delta N /{ }^{\prime}$. & $\Delta V / \cdot \Delta$ & $r / I F$ \\
\hline NN & تعداد گُره & r/AD & T/Va & r/gr \\
\hline FLAn & زاويه برگ يرجم & $r$ rev & שום/4r & $-1 / 91$ \\
\hline LFIN & طول ميانگره اول از انتها (سانتىمتر) & $19 / \cdot 1$ & $|N|$ & $1 / 19$ \\
\hline
\end{tabular}

1000GW= 1000-grain weight; GY = Grain yield; SY= Straw yield; BY= Biological yield; HI= Harvest index; NGS= Number of grains per spike; NFT= Number of fertile tiller; FLL= Flag leaf length; FLW = Flag leaf width; FLAr= Flag leaf area; DS= Days to $50 \%$ heading; DF= Days to $50 \%$ flowering; height; NN= Number of nods; FLAn= Flag leaf angle; LFIN= Length of the first inter node from top.

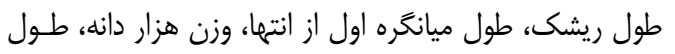

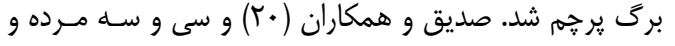

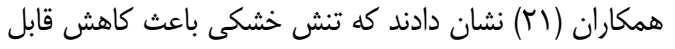

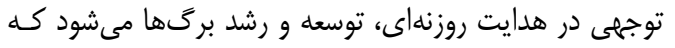

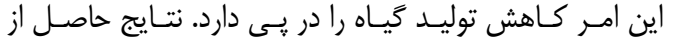

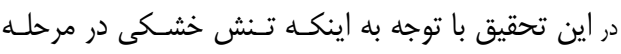

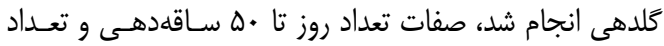

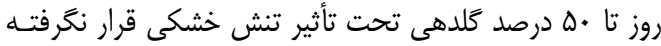

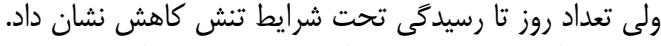

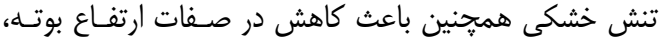




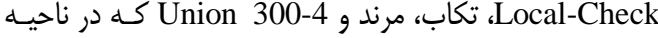

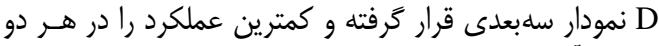

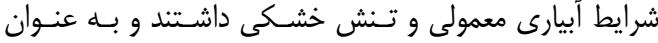

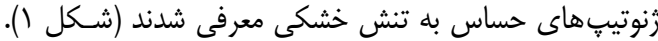

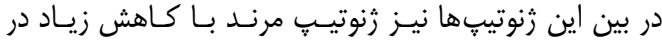

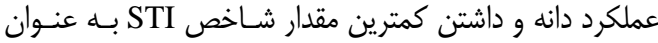

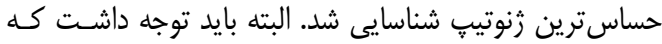

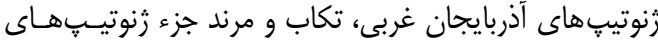

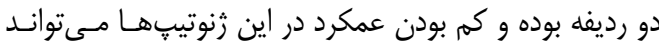
ناشى از اين صفت باشد.
مقايسه ميانخينها (جدول ه) نشان داد كه در شـرايط آبيـارى

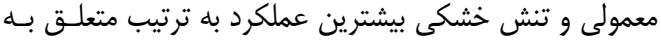

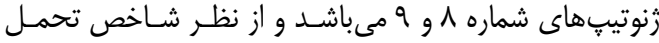

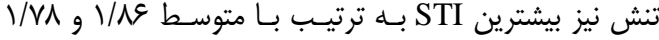

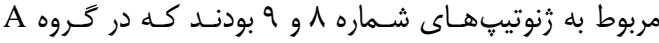

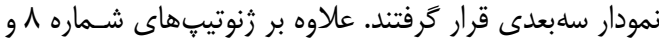

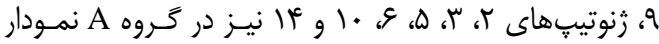

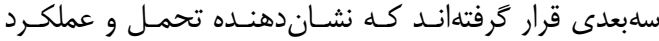

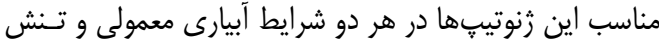

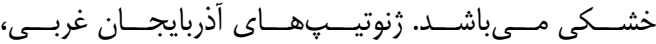

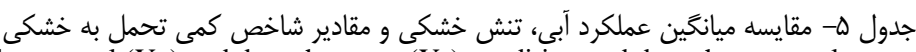
Table 5. Mean compression of yield under normal (Yp) and drought stress (Ys) condition and drought stress tolerance

\begin{tabular}{|c|c|c|c|c|}
\hline STI & Ys & Yp & اسم زنوتيب & شماره زنوتيت \\
\hline A ג & $V \mu / r \cdot{ }^{h}$ & $\mathrm{~V} Q / / \varepsilon \mathrm{V}^{\mathrm{h}}$ & Local- Check & 1 \\
\hline ./arre & $|r| \cdot / \Delta V^{\mathrm{de}}$ & $19 \mathrm{r} / . .{ }^{\text {cde }}$ & Rihane & r \\
\hline ( & $\mid r \Delta V / \mu^{C d}$ & $r \mid f y / / r^{b c}$ & Rihane/ Alger- Union & r \\
\hline .19919 & $\| \digamma \mathcal{N} / \mathrm{He}_{\mathrm{de}}$ & (rی)/ & Atlas 46/ Kavir & f \\
\hline I/TVIT & $\mid f \wedge \cdot / .^{b}$ & $r \cdot \Delta V / F V^{c d}$ & Gorgan/ CM 67/ Pro/ Svo & $\Delta$ \\
\hline r & $q \vee \diamond / . . \mathrm{fg}$ & IVTN/Me & Suifu/ Walfajre & 4 \\
\hline.$/$ VITY & $\| \varepsilon 9 / . \mathrm{de}$ & $\mid f \& \& / F V^{f}$ & $\mathrm{QC} 2.17 / \mathrm{D} 7 / \mathrm{Bgs}$ & $\mathrm{v}$ \\
\hline V/AGT & $|V+1 / /|^{a}$ & $r \Delta S Y / V^{\mathrm{a}}$ & Kavir/ Badia & $\wedge$ \\
\hline I/VAFA & $|A F| / g V^{a}$ & rMr/A. ${ }^{b}$ & Rihane- O5 & 9 \\
\hline$\cdot / v \cdot v r$ & $q \neg q / \kappa r^{g}$ & $\mid \Lambda r r / r \cdot{ }^{\text {de }}$ & AS 46/ Ahtaz- ZAD & 1. \\
\hline.$/ T Y Y \Lambda$ & $\operatorname{V\Lambda r/l} \cdot{ }^{h}$ & $\Lambda r \Delta / \wedge^{h}$ & Landrace (West Azarbaijan) & 11 \\
\hline.$/ M \cdot \Lambda$ & $\Delta V Q / \kappa r^{i}$ & $v^{*} v / v v^{h}$ & Landrace (Tekab) & Ir \\
\hline .1 .991 & $r q \tau / q^{j}$ & h hr/rh & Landrace (Marand) & זו \\
\hline $1 / \cdot 1 \mathrm{VI}$ & brec & $|V \Lambda| / \cdot v^{e}$ & Hebe & if \\
\hline 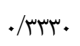 & $9 \wedge / 1 .{ }^{\mathrm{hi}}$ & $\| G T / V^{g}$ & Union $300-4$ & 10 \\
\hline.$/ 9 \Delta V \Delta$ & $11 \ldots /{ }^{e f}$ & ff & Landrace (Salmas) & 18 \\
\hline
\end{tabular}

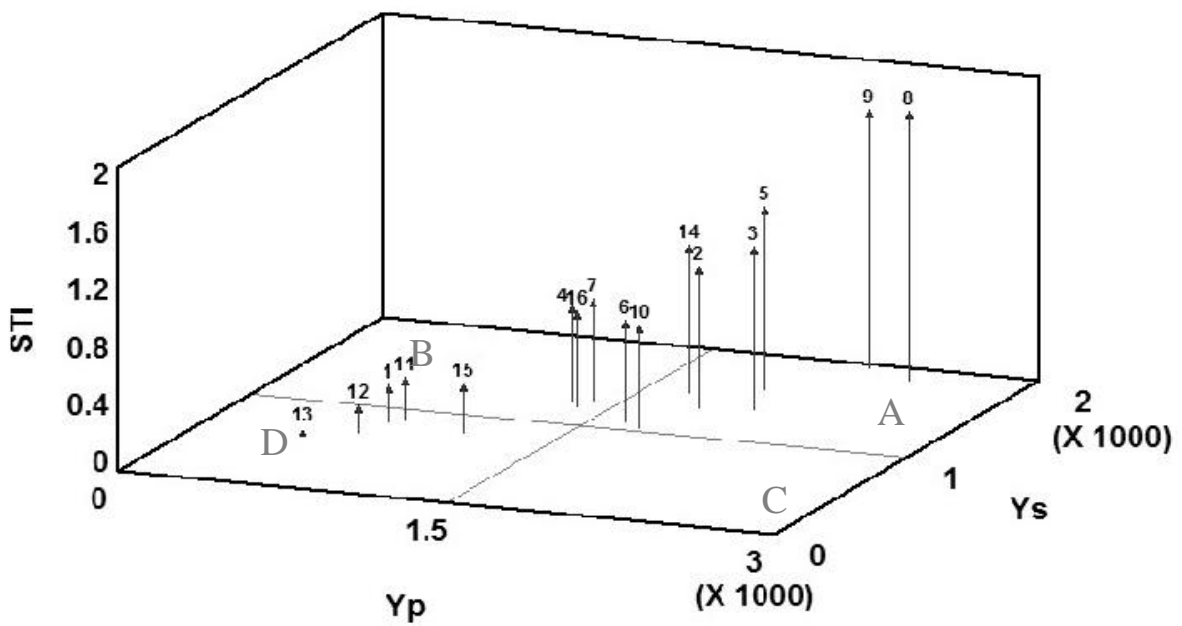

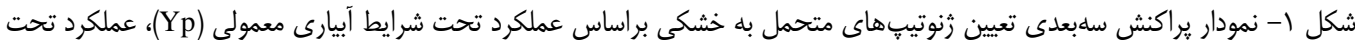

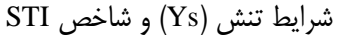

Figure 1.3D plot for determination tolerant genotypes to drought stress based on Yp, Ys and STI index 1= Local- Check; 2= Rihane; 3= Rihane/ Alger- Union; 4= Atlas 46/Kavir, 5= Gorgan/ CM 67/ Pro/ Svo; 6= Suifu/ Walfajre; 7= QC 2.17 /D7/ Bgs; 8= Kavir/ Badia; 9= Rihane- O5; 9= AS 46/ Ahtaz- ZAD; 11= Landrace (West Azarbaijan); 12= Landrace (Tekab); 13= Landrace (Marand); 14= Hebe; 15= Union 300-4; 16= Landrace (Salmas). 


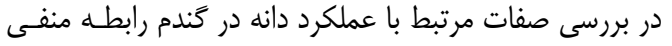

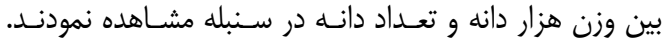

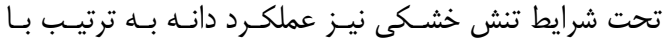

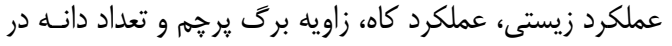

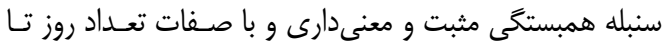

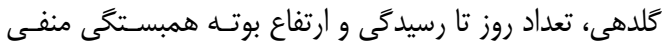

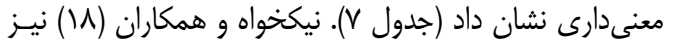

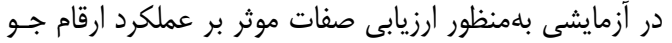

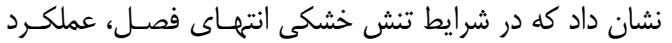

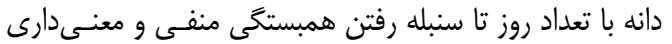

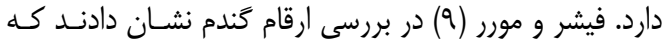

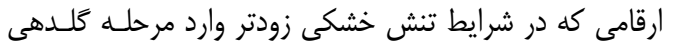

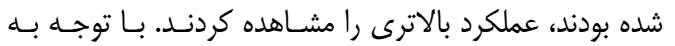

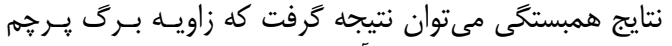

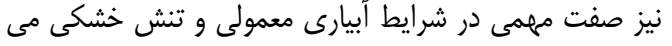

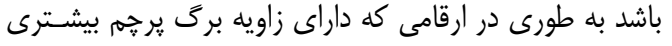

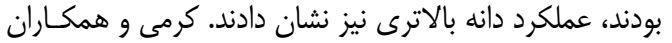

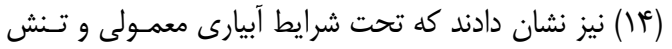

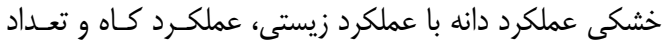

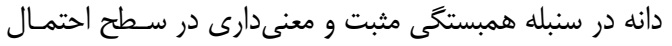

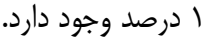

تعيين ضرايب هبستتى ساده إه

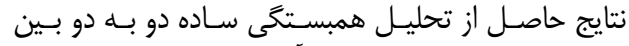

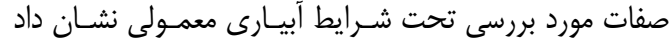

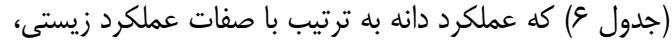

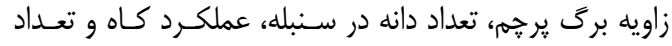

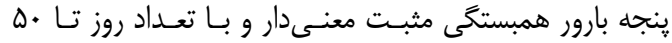

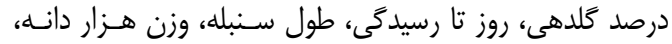

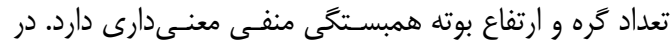

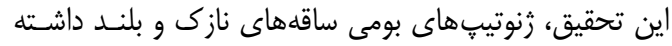

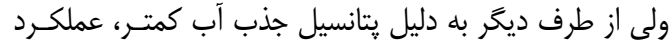

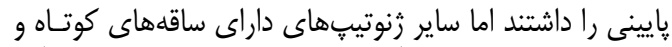

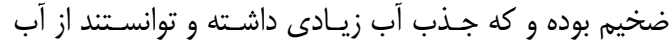
آبيارى در جهت توليد محصول بيشتر استفاده كنند. تعداد دانسه

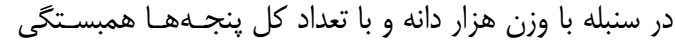

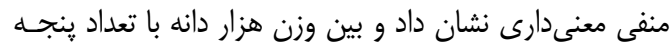

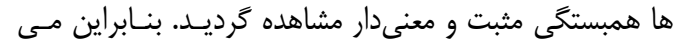

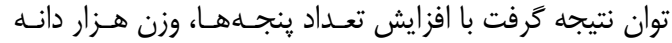

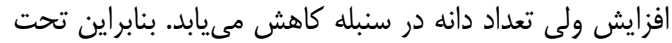

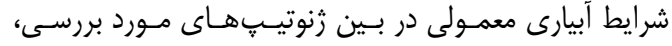

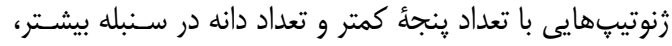

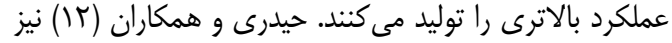


Table 6. Correlation coefficients between investigated traits of barley genotypes under normal conditions جدول ع- همبستى بين صفات مورد بررسى در عا زنوتي جو تحت شرايط آبيارى معمولى

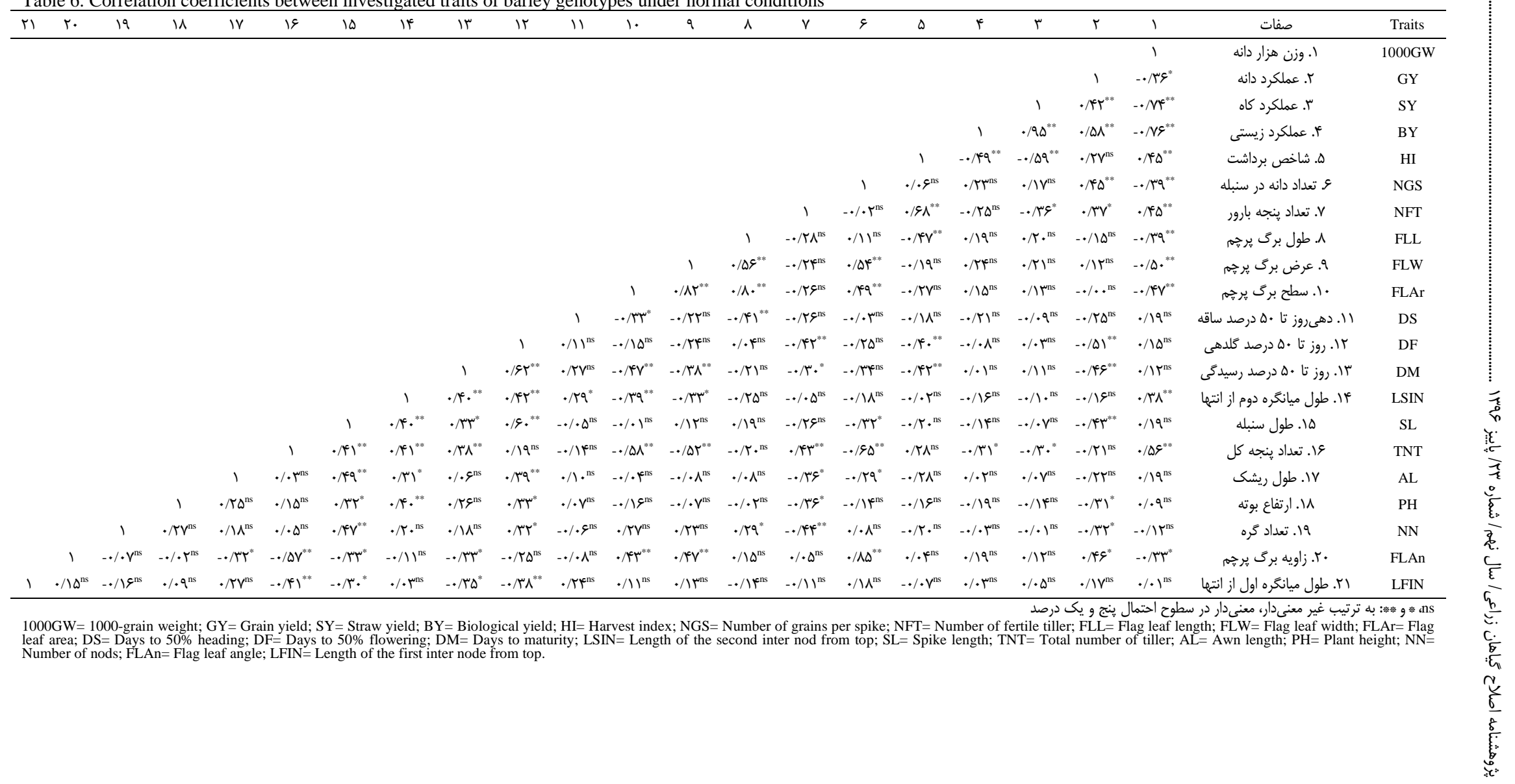




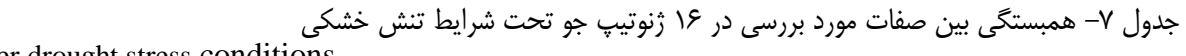

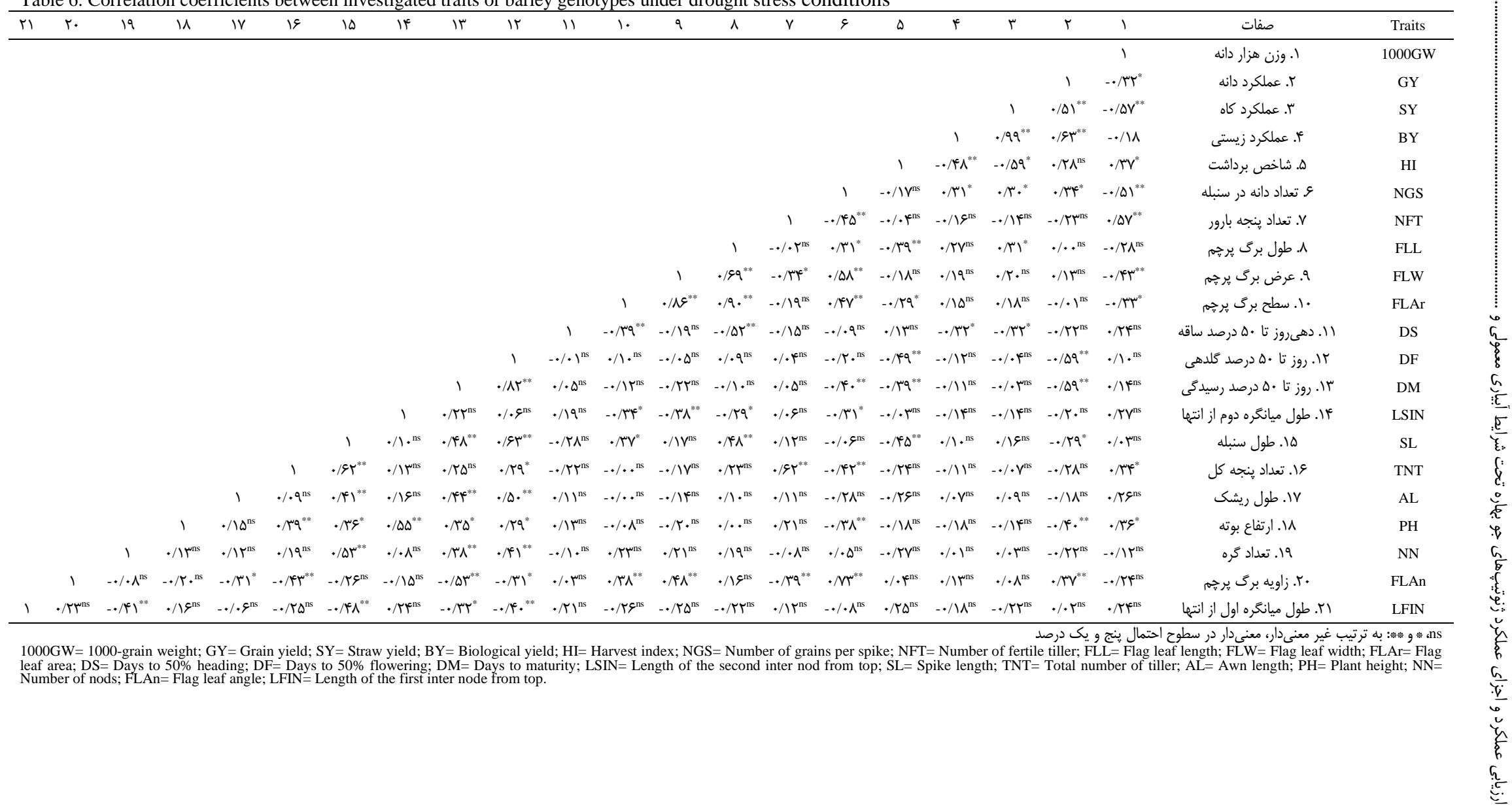




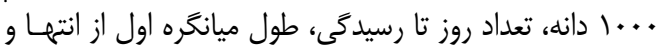

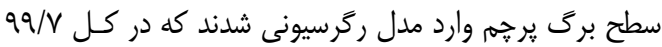

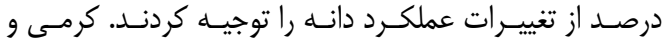

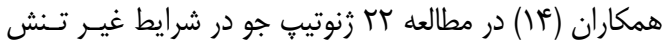

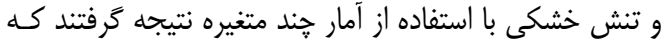

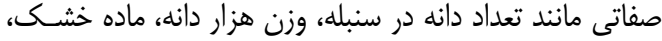

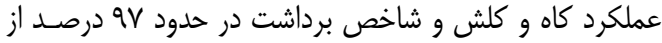

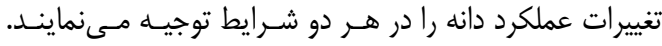

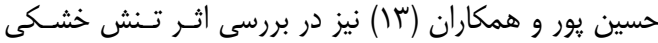

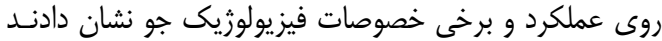

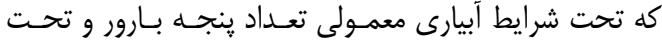

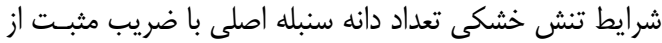

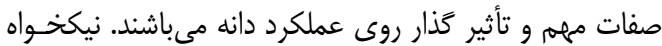

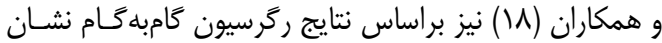

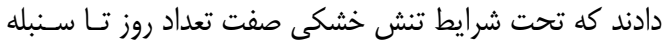

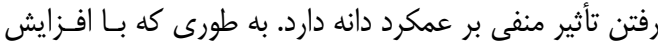

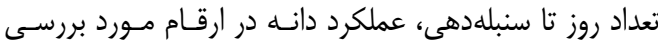

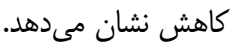

تجزيه رَّرسيون كَامبه

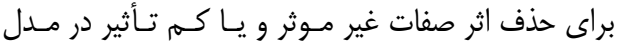

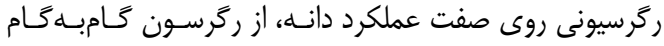

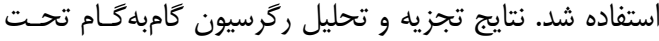

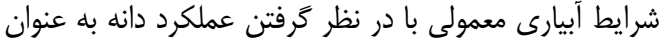

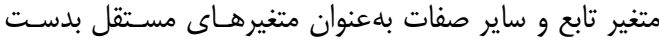

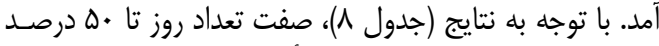

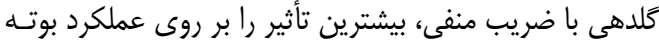

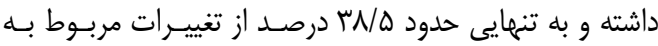

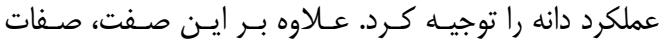

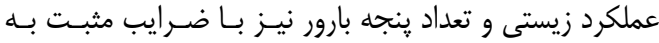

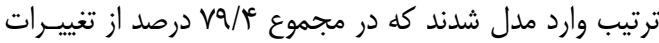

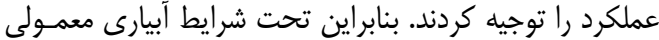

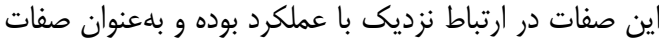

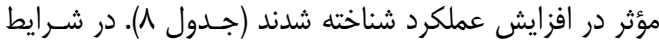

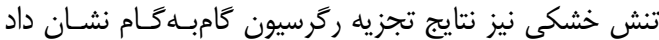

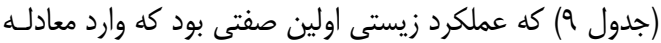

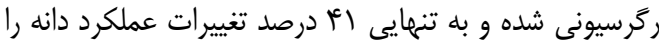

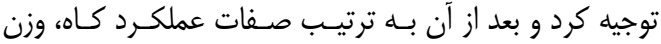

جدول ^- نتايج رگرسيون گامبهَام بين عملكرد دانه با ساير صفات مورد بررسى در عا زنوتيب جو تحت شرايط آبيارى معمولى Table 8. Stepwise regression analysis for seed yield and other investigated traits in sixteen barley genotypes under

\begin{tabular}{|c|c|c|c|c|}
\hline $\mathrm{R}^{r}$ & مقدار برآورد شده & صفات & Traits & مرحله \\
\hline- & $19 \Lambda \rho / \% \Lambda^{\mathrm{ns}}$ & عرض از مبدأ & Intercept & - \\
\hline 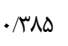 & $-\mu \psi / \lambda V F^{\mathrm{ns}}$ & تعداد روز تا •ه درصد كلدهى & DF & 1 \\
\hline .1909 & (r.**** & عملكرد زيستى & BY & $r$ \\
\hline . NqF & $V r \cdot / \Delta \Lambda .^{*}$ & تعداد ينجه بارور & NFT & r \\
\hline
\end{tabular}

$\mathrm{DF}=$ Days to $50 \%$ flowering; $\mathrm{BY}=$ Biological yield; $\mathrm{NFT}=$ Number of fertile tiller

جدول q- نتايج ركرسيون كامبه كام بين عملكرد دانه با ساير صفات مورد بررسى در عا زُنوتيب جو تحت شرايط تنش خشكى Table 9. Stepwise regression analysis for seed yield and other investigated traits in sixteen barley genotypes under

\begin{tabular}{|c|c|c|c|c|}
\hline $\mathrm{R}^{r}$ & مقدار برآورد شده & صفات & Traits & 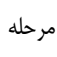 \\
\hline- & rqme/sq** & عرض از مبدأ & Intercept & - \\
\hline.$|4|$ & $\cdot / v 8^{* *}$ & عملكرد زيستى & BY & 1 \\
\hline אי &.$- / v q^{* *}$ & عملكرد كاه & SY & r \\
\hline . & $-\mid r / 1^{* *}$ & وزن هزار دانه & $1000 \mathrm{GW}$ & r \\
\hline.$/ 9 \vee \Delta$ & - & تعداد روز تا •ه درصد رسيدگى & DM & r \\
\hline.$/ 919$ & $-\kappa \varphi / . . * *$ & طول ميانكره اول از انتها & LFIN & $\Delta$ \\
\hline.$/ 998$ & $-9 / \cdot 9^{* *}$ & سطح برك يرجم & FLAr & 8 \\
\hline
\end{tabular}

BY= Biological yield; SY= Straw yield; 1000GW= 1000-grain weight; DM= Days to maturity; LFIN= Length of the first inter node from top; FLAr= Flag leaf area.

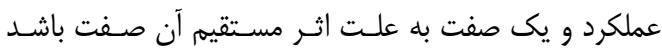

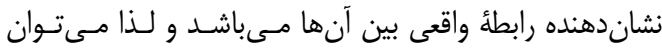

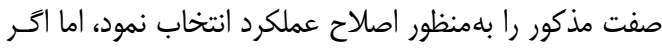

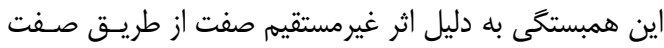

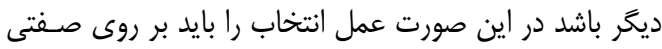

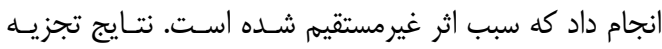

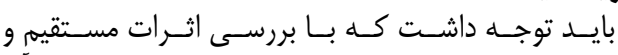

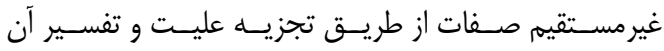

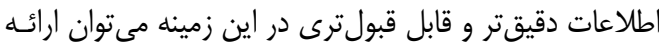

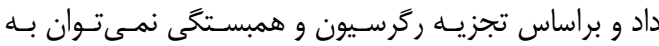

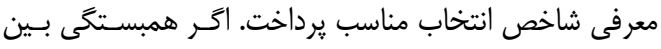




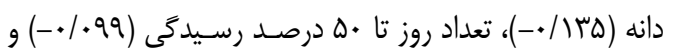

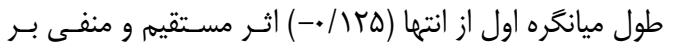

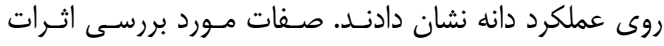

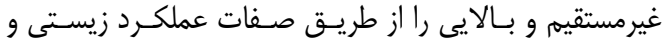

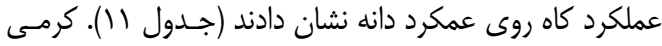

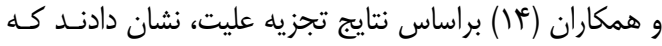

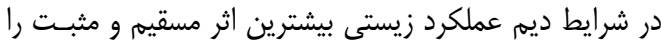

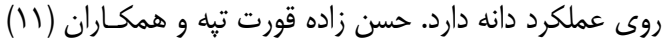

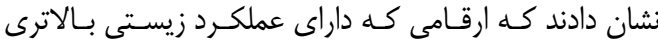

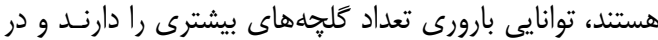

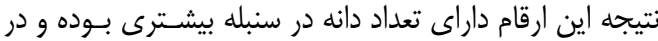

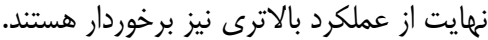

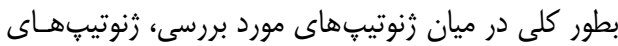
Kavir/Badia

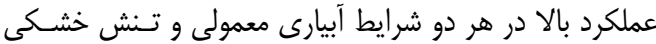

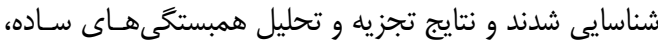

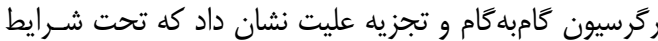

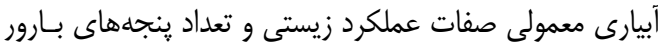

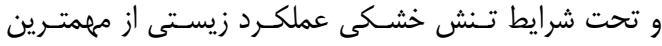

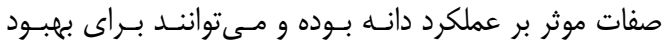

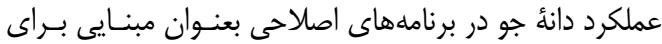
انتخاب مورد توجه قرار كيرند.
عليت تحت شرايط آبيارى معمولى نشان داد (جـدول • •(1) كـهـ

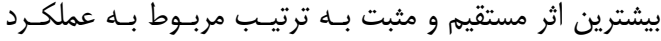

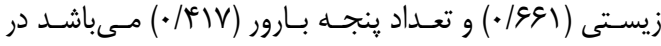

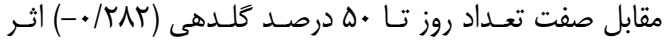

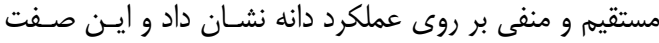

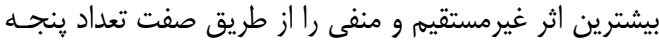

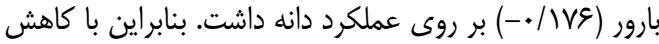

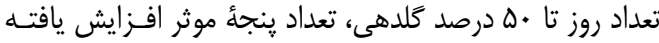

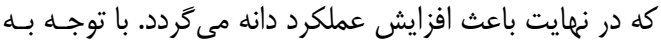

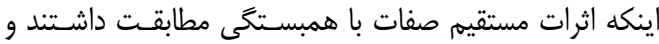

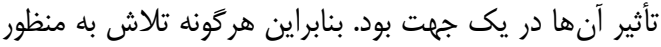

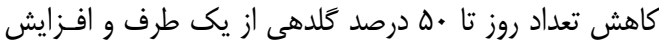

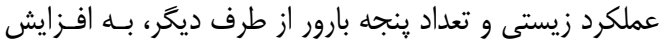

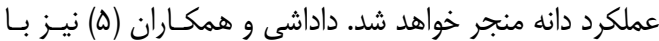

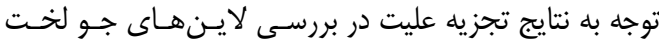

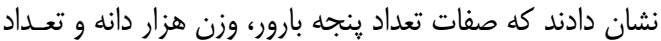

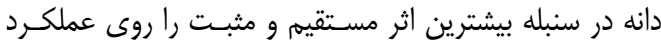

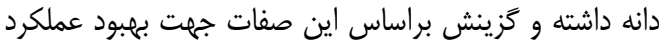

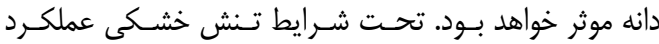

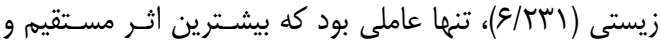

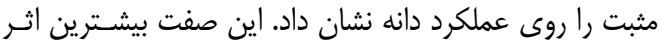

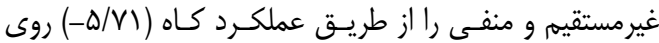

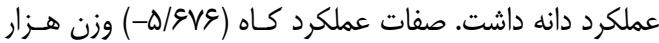

جدول • ا- تجزيه ضرايب عليت براى عملكرد دانه در عا زُنوتيب جو تحت شرايط آبيارى معمولى Table 10. Path coefficient analysis for grain yield of sixteen barley genotypes under normal conditions

\begin{tabular}{|c|c|c|c|c|c|c|}
\hline \multicolumn{3}{|c|}{ اثر غير مستقيم از طريق } & \multirow[t]{2}{*}{ اثر مستقيه } & \multirow{2}{*}{ ضريب همبستكى } & \multirow{2}{*}{ صفات } & \multirow{2}{*}{ Traits } \\
\hline r & r & 1 & & & & \\
\hline.$- / 1 V 9$ & $-\cdot / \cdot \Delta r$ & - & $-\bullet / T \wedge T$ &.$- / D 1$ & تعداد روز تا •ه درصد كلدهى & DF \\
\hline$\because / 1 \cdot \omega$ & - &.$\% 4 t$ & .1991 &.$/ 01$ & عملكرد زيستى & BY \\
\hline- &.$- / 199$ &.$/ 11$ & . אIV & $\cdot / r r$ & $\begin{array}{c}\text { تعداد ينجه بارور } \\
\text { Residual= } 0.564\end{array}$ & NFT \\
\hline
\end{tabular}

$\mathrm{DF}=$ Days to $50 \%$ flowering; $\mathrm{BY}=$ Biological yield; NFT $=$ Number of fertile tiller.

جدول ||- تجزيه ضرايب عليت براى عملكرد دانه در 1 إزنوتيب جو تحت شرايط تنش خشكى

Table 11. Path coefficient analysis for grain yield of sixteen barley genotypes under drought stress conditions

\begin{tabular}{|c|c|c|c|c|c|c|c|c|c|}
\hline \multirow[b]{2}{*}{9} & \multicolumn{5}{|c|}{ اثر غير مستقيم از طريق } & \multirow[t]{2}{*}{ اثر مستقيم } & \multirow{2}{*}{ ضريب همبستگى } & \multirow{2}{*}{ صفات } & \multirow{2}{*}{ Traits } \\
\hline & $\Delta$ & f & r & r & 1 & & & & \\
\hline$\%$ &.$- / . t r$ & $\cdot / \cdot 1$ & $\cdot / \cdot V^{c}$ & $-\Delta / V)$ & - & ( & سצ/. & عملكرد زيستى & BY \\
\hline$\%$ &.$/ \cdot \mathrm{TV}$ & 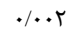 & $\cdot / \cdot V 9$ & - & g/ & $-\Delta / V \& V$ & $\cdot|\Delta|$ & عملكرد كاه & SY \\
\hline$-*+t$ &.$-++r$ & $-* / .1 f$ & - & T/TAV & 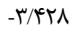 & 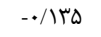 & 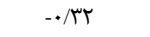 & وزن هزار دانه & $1000 \mathrm{GW}$ \\
\hline$-* / *+1$ &.$/ .49$ & - &.$- .1 \cdot 19$ &.$/ \mathrm{Vr}$ & $-* / 9 \wedge 9$ &.- .1 .99 & $-* 109$ & تعداد روز تا •ه درصد رسيدگى & DM \\
\hline$-*+* r$ & - & ( ) & س & $1 / T \& \Lambda$ & -I/ITK & 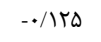 & $\cdot / \cdot r$ & طول ميانخره اول از انتها & LFIN \\
\hline - & t. & $\cdot 1 \cdot 11$ & $.1 \cdot e^{4}$ & $-1 / \cdot r q$ &.$/ 9 M F$ & $\%+$ & $\cdot /-\cdot 1$ & $\begin{array}{l}\text { سطح برگ يرخه } \\
\text { Residual= -0.083 }\end{array}$ & FLAr \\
\hline
\end{tabular}


منابع 18: 31-33.

2. Aspinall, D. 1965. The effects of soil moisture stress on the growth of barley I. Vegetative development and grain yield. Australian Journal of Agricultural Research, 15: 729-745.

3. Bhutta, W.M., T. Barley and M. Ibrahim. 2005. Path-coefficient analysis of some quantitative characters in husked barley. Caderno de Pesquisa Ser Biol, 17: 65-70.

4. Dadashi, M.R. 2006. Effect of salinity stress on morphophisiological important agronomic characteristic barley and hull-less barley cultivars. Thesis for degree of Ph.D in Plant Breeding. Islamic Azad University, Research and Science Branch, 90 pp (In Persian).

5. Dadashi, M.R., A.A. Nouri Nia, M. Asgar and Sh. Azizi Chakherchaman. 2010. Evaluation of correlation between yield and some of physiological and morphological traits in nude barley variety. Journal of Crop and Weed Eco-physiology, 15: 29-40.

6. Debaeke, P. and A. Abdellah. 2004. Adaptation of crop management to water-limited environments. European Journal of Agronomy, 21: 433-446.

7. FAO. 2010. Statistical data. www. FAOSTAT. Org.

8. Fernandez, G.C.J. 1992. Effective selection criteria for assessing plant stress tolerance. In: Proceeding of a symposium, Taiwan, 13-18 August, 257-27 pp.

9. Fisher, R.A. and R. Maurer. 1978. Drought resistance in spring wheat cultivars I. Grain yield responses. Australian Journal of Agricultural Research, 29: 897-912.

10. Fowler, C.W. and D.C. Rasmusson. 1969. Leaf area relationships and inheritance in barley. Crop Sci, 9: 729-731.

11. Hassanzadeh Gorttapeh, A., A. Fathollahzadeh, A. Nasrollahzadeh Asl and N. Akhondi. 2008. Agronomic nitrogen efficiency in different wheat genotypes in west Azerbijan province. Electronic Journal of Crop Production, 1: 82-100.

12. Heydari, B., G. Saeedi and B.A. Seyed Tabatabai. 2007. Factor analysis for quatitative traits and evaluation of path analysis for grain yield in wheat. Journal of Science and Technology of Agriculture and Natural Resources, 42: 135-143 (in Persian).

13. Hossein Pour, J., M. Kafi and M.J. Mir Hadi. 2008. Effect of drought stress on yield and some of physiological traits in barley. Iranian Journal of Agricultural Science, 39: 165-177.

14. Karami, E., M.R. Ghannadha, M.R. Naghavi and M. Mardi. 2005. An evaluation of drought resistance in barley. Iranian Journal of Agricultural Science, 36: 547-560.

15. Koocheki, A. 1994. Crop production in dry region: Cereals, Legumes, Industrial and forage crops (Translated in Persian). Jihad Daneshghahi Mashhad Press. 202 pp.

16. Najeeb, S. and S.A. Wani. 2004. Correlation and path analysis studies in barley (Hordeum vulgare L.). National Journal of Plant Improvement, 6: 124-125.

17. Nazari, L. and H. Pakniyat. 2008. Genetic diversity of wild and cultivated barley genotypes under drought stress using RAPD markers. Biotechnology, 7: 745-750.

18. Nikkhah, H.R., M.H. Saberi and M. Mahlouji. 2010. Study of effective traits on grain yield of two and six row barley genotypes (Hordeum vulgare L.) under terminal drought stress conditions. Iranian Journal of Crop Sciences, 12: 170-184 (In Persian).

19. Omara, M.K. 1987. Selection of early maturing barley with improved response to drought stress. Australian Journal of Agricultural Research, 38: 835-845.

20. Siddique, M.R.B., A. Hamid and S. Islam. 1999. Drought stress effect on photosynthetic rate and leaf gas exchange of wheat. Botanical Bulletin- Academia Sinica Taipei, 40: 141-145.

21. Siosemardeh, A., A. Ahmadi, K. Poustini and H. Ebrahimzadeh. 2003. Stomatal and nonstomatal limitations to photosynthesis and their relationship with drought resistance in wheat cultivars. Iranian Journal of Agriculture Science, 34: 93-106.

22. Tomer, S.B. and G.H. Prasad. 1999. Path coefficient analysis in barley. Department of Agricultural Botany, S.D.J. Pos Graduate College Chandesar Azamgarh, 27: 28-61.

23. Vaisi Mal Amiri, V., R. Hagh Parast, M.E. Aghaee Sarbarzeh, A. Farshadfar and R. Rajabi. 2010 Evaluation of drought tolerance in barley genotypes (Hordeum vulgare L.) using physiological characteristics and indicators of drought tolerance. Seed and Plant Journal, 26: 43-60. 


\title{
Evaluation of Yield and Yield Component of Spring Barely Genotypes under Late Season Drought Stress
}

\author{
Ali Soleimani ${ }^{1}$, Mostafa Valizadeh ${ }^{2}$, Reza Darvishzadeh ${ }^{3}$, Saeed Aharizad ${ }^{4}$ and \\ Hadi Alipour ${ }^{5}$ \\ 1- PhD Student, Faculty of Agriculture, Science and Research Branch, Islamic Azad University \\ 2 and 4- Professor and Associate Professor, Faculty of Agriculture, University of Tabriz \\ 3- Professor, Urmia University \\ 5- Assistant Professor, Urmia University \\ (Corresponding author: alipourhadi64@gmail.com) \\ Received: March 12, 2016 \\ Accepted: July 4, 2016
}

\begin{abstract}
In order to evaluation of gain yield and yield components in spring barely genotypes under normal and drought stress in flowering stage and identifying drought tolerant genotypes, sixteen barely genotypes were investigated in two separately Randomized Complete Block Design with three replications in West Azerbaijan Agricultural and Natural Resources Research Center, Urmia, Iran in agronomic season at 2011-2012 crop season. Result of analysis of variance showed that there are highly significant differences among genotypes in studied traits which reveal high genetic variation among the genotypes that provide a choice possibility based on studied traits. Based on the results of mean compression under normal and drought stress condition and drought tolerance index (STI), Kavir/Badia and Rihane-O5 genotypes were introduced as tolerant genotypes with high grain yield and local west Azerbaijan, Local-Check, Tekab and Marand genotypes were introduced as drought-sensitive genotypes with low yield. According to the results of simple correlations analysis, stepwise regression and path analysis in normal irrigation conditions, biological yield and number of fertile tiller and in drought stress condition biological yield were the most important of effective traits on grain yield. Since, the biological yield had high positive direct effect on grain yield under normal and drought stress condition, it can be one of the most important and stable traits for grain yield improvement in the breeding programs.
\end{abstract}

Keywords: Barley, Correlation analysis, Drought stress, Path analysis, Stepwise regression 